\title{
Phenomenological approach to mechanical damage growth analysis
}

\author{
Nicola Pugno, ${ }^{1}$ Federico Bosia, ${ }^{2}$ Antonio S. Gliozzi, ${ }^{2}$ Pier Paolo Delsanto, ${ }^{2}$ and Alberto Carpinteri ${ }^{1}$ \\ ${ }^{1}$ Department of Structural Engineering and Geotechnics, Politecnico di Torino, Corso Duca degli Abruzzi 24, 10129 Torino, Italy \\ ${ }^{2}$ Department of Physics, Politecnico di Torino, Corso Duca degli Abruzzi 24, 10129 Torino, Italy \\ (Received 27 February 2008; revised manuscript received 16 June 2008; published 3 October 2008)
}

\begin{abstract}
The problem of characterizing damage evolution in a generic material is addressed with the aim of tracing it back to existing growth models in other fields of research. Based on energetic considerations, a system evolution equation is derived for a generic damage indicator describing a material system subjected to an increasing external stress. The latter is found to fit into the framework of a recently developed phenomenological universality (PUN) approach and, more specifically, the so-called U2 class. Analytical results are confirmed by numerical simulations based on a fiber-bundle model and statistically assigned local strengths at the microscale. The fits with numerical data prove, with an excellent degree of reliability, that the typical evolution of the damage indicator belongs to the aforementioned PUN class. Applications of this result are briefly discussed and suggested.
\end{abstract}

DOI: 10.1103/PhysRevE.78.046103

PACS number(s): 62.20.mm, 02.60.Gf, 07.05.Tp, 05.10.-a

\section{INTRODUCTION}

It is well known that the mechanisms governing the evolution of damage and fracture in solids may vary greatly, depending on the kind of materials considered, their degree of disorder, the size scale involved, the type of applied loading, etc. [1]. However, regardless of the mechanisms occurring at the microscale and of the specific values of the elastic parameters, some ubiquitous elements recur in the overall macroscopic behavior. Indeed, similar patterns of acoustic emission activity, stress-strain relationship, or nonlinear hysteretical behavior can be observed in very different material specimens ([2] and references therein). This is also true for the observed scaling properties of other quantities, such as the dissipated energy or the material strength, for which a fractal geometrical dependence seems to have been ascertained [3]. In fact, the overall macroscopic behavior of systems undergoing damage seems to emerge from phenomena occurring at the microscale, which are also typical of other physical systems that are driven out of equilibrium (e.g., Barkhausen noise in ferromagnets [4]) and can therefore be treated with methods typical of statistical mechanics [5]. More in general, one would expect strong similarities to exist between the damage evolution in materials and other selflimiting growth phenomena observed in other contexts, where the macroscopic growth process is governed by a statistical ensemble of smaller-scale units. Examples outside the area of physics or material science could be the individual cells in the case of ontogenetic growth or individual settlements in the case of urban growth (for which the evolution equations may be derived from energetic considerations [6]).

A considerable amount of effort has been devoted in past years to the description of those systems occurring in such diverse fields as physics, biology, statistics, and economics that undergo a time evolution that includes a growth phase often followed by saturation. Historically, well-established models, such as those based on the logistic [7], Von Bertalanffy [8], or Gompertz [9] equations, have been often applied and modified according to the specific problems to be studied. More recently, the allometric growth model of West and collaborators [6] has stimulated further interest in the analysis of the mechanisms responsible for growth and in the search for common characteristics between systems, which are very different in both scale and context $[10,11]$. This has led to the development of very interesting concepts, such as the formulation of life's universal scaling laws [12]. An extension of West's law to neoplastic growths has been also suggested by Delsanto and collaborators $[13,14]$, in which a dynamical evolution of the fractal exponent is proposed to describe the transition from an avascular to an angiogenetic stage of the tumor growth. These studies have led to the development of a phenomenological universality (PUN) "bottom-up" approach [15], which seeks to describe an almost endless variety of self-limiting growth problems, independently of the field of application.

In this paper we aim to derive equations that should apply to a generic damage evolution model and to compare them with previous growth models (derived in the framework of a top-down approach). We then exploit a simple numerical model to simulate a typical damage progression experiment on a generic material sample and obtain the macroscopic behavior from a statistical ensemble of microscopic elements. The synthetic data are then fitted using the PUN formalism. This procedure allows the determination, with a high degree of confidence, of a correspondence between damage growth problems and other phenomena belonging to the same PUN class, possibly leading to some cross-fertilization among apparently totally unrelated phenomenologies.

\section{DAMAGE GROWTH MODEL}

Let us consider an unspecified physical system subjected to an external stress that is increasing in time. In fact, the analysis presented in this section can be extended to other systems in diverse fields, where some kind of "damage indicator" $Y$, as well as a generic "external stress" $\sigma$ causing the damage can be identified [16,17]. For example, we could consider a solid having a volume $V$ with a growing damaged subvolume portion $v$, due to an increasing external stress $\sigma$. 
Thus, we would define $Y=v / V$ and write the damage evolution equation in the classical form $[18,19]$

$$
\frac{d Y}{d t}=g(Y, \sigma) .
$$

During the damage process, the power loss by both fracture and friction can be written as $W=w v^{p}[3,20]$, where $p$ $=D / 3$ and $D$ is the fractal dimension [21] of the dissipation domain $(2<D<3)$ and $w$ is the power loss per "fractal volume." Indeed, it has been often observed that the damage process occurs in a fractal domain comprised between that of a surface and of a volume, with size-dependent multifractal exponent $D$, ranging from 2 for small sizes to 3 for large sizes. Rare cases with $D>3$ (e.g., for asteroids $D$ is around 4) have also been observed in the fracture of threedimensional solids (this seems to be a consequence of a particle size distribution with $D>3$ [22]).

The conservation of energy implies

$$
W=f v+F d v / d t
$$

since the energy is dissipated both as friction $(f)$ on preexisting defects and in fracture $(F)$, to create new defects. Accordingly, by dividing (2) by $V$ and rearranging, we may describe the growth of fractal damage as

$$
\frac{d Y}{d t}=\frac{w}{F V^{1-p}} Y^{p}-\frac{f}{F} Y .
$$

Since it is reasonable to assume that this type of physical system belongs to the category of self-limiting growth phenomena (but this condition can be straightforwardly eliminated), we impose the saturation of the damage factor (i.e., $d Y / d t=0$ at $Y=1)$, so that $w=f V^{1-p}$. Accordingly,

$$
\frac{d Y}{d t}=\frac{f}{F}\left(Y^{p}-Y\right)
$$

This growth law is identical to Eq. (9) in [14], which is the characteristic equation for the PUN class U2, provided

$$
\alpha_{2}=\beta_{2}=\frac{f}{F} .
$$

It is interesting to note the analogy between this system and other self-limiting growth phenomena, where the evolution equations are determined by the balance between competing processes. Fracture and friction in this case play the role of, e.g., cell growth and maintenance.

Integrating Eq. (4), the evolution of the damage can consequently be described by

$$
Y(t, \sigma)=\left[1-\left(1-Y_{0}^{1-p}\right) e^{-(f / F)(1-p) t}\right]^{1 /(1-p)},
$$

where $Y_{0}=Y(0, \sigma)$ and where we used $Y_{\infty}=\lim _{t \rightarrow \infty} Y(t, \sigma)$ $=1$.

In the case of a linearly increasing applied stress $\sigma=k t$, Eq. (6) can also be seen as a damage evolution equation with respect to stress. This dependence is visualized in Fig. 1. We have chosen for simplicity the following set of parameters: $f=1 \mathrm{~J} / \mathrm{s}, F=1 \mathrm{~J}$, and $k=2 \times 10^{6} \mathrm{~Pa} / \mathrm{s}$. Four different curves are presented, for $p=0.1,0.6,0.8$, and 0.9. Two different

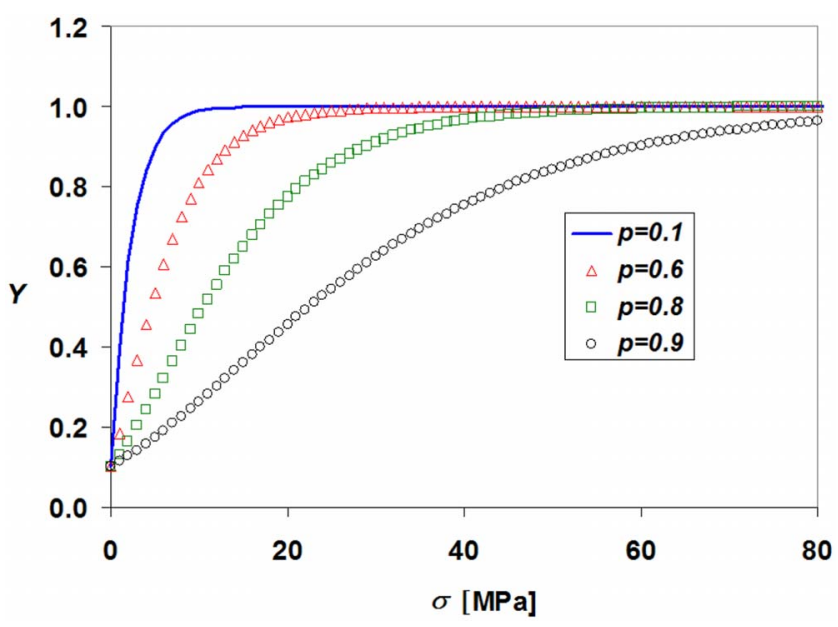

FIG. 1. (Color online) Damage factor $(Y)$ dependence on increasing stress $\sigma$ for increasing values of the fractal exponent $p$.

types of growth curves (logistic- or sigmoid-like) can occur for different $p$ values, depending on whether inflection points exist for $\sigma>0$ or not.

Turning to the time dependence expressed in Eq. (6), the model predicts a maximum damage rate $\left(d^{2} Y / d t^{2}=0\right)$ before failure (as observed during acoustic emission) at $t^{*}$ $=-\frac{F}{f} \ln \left[\frac{1-p}{1-Y_{0}^{1-p}}\right]$, with $Y^{*}=p^{1 /(1-p)}$-i.e., as a function of the fractal dimension only. In fact, $Y^{*}$ could represent a "critical damage level". Defining the failure at a conventional value of the damage level (e.g. percolation threshold) $Y^{*} \leqslant Y_{f}<1$ one can derive the time to failure $t_{f} \geqslant t^{*}$, since $Y(\infty, \sigma)=1$.

\section{NUMERICAL SIMULATIONS}

In order to verify the applicability of the model proposed in the previous section, we have generated synthetic data using a recently developed simulation code for the description of damage progression and acoustic emission (AE) in materials [23] and verified the correlation of the results with the U2 class. The simulation code is based on an equal-loadsharing fiber-bundle-model (FBM) approach [24-28], with randomly assigned (Weibull-distributed) fiber strengths $\sigma_{C i j}$. The specimen is modeled by adopting a discretization in $N_{x}$ "bundles" of $N_{y}$ fibers and by applying at every time step the analytically calculated local loads deriving from an increasing externally applied stress $\sigma(t)$. An AE event is generated whenever the local stress exceeds the assigned fiber peak stress. In this case the corresponding fiber stiffness $k_{i j}$ is set to zero and the related section (or bundle) undergoes a corresponding stiffness reduction. This can be considered as an example of a model with quenched disorder. An alternative approach is to adopt a model with annealed disorder by decreasing adjacent fiber strengths in correspondence with fiber ruptures. However, this modification is beyond the scope of this work and will be discussed elsewhere.

Energy dissipation owing to the formation of new fracture surfaces at each AE event is also accounted for in the formulation: energy balance requires that the variation of the total potential energy $\Delta U_{i j}(t)$, when an AE event occurs at the 
location $(i, j)$, be compensated by the kinetic energy $\Delta T_{i j}(t)$, released in the form of a stress wave, and dissipated energy $\Delta \Omega_{\iota j}(t)$, in the formation of a crack surface at micro- or mesoscale. Thus, we can write

$$
\Delta U_{i j}(t)+\Delta T_{i j}(t)+\Delta \Omega_{i j}(t)=0 .
$$

The potential energy variation $\Delta U_{i j}(t)$ is related to the imposed displacement $x(t)$ (or force) and the overall specimen stiffness variation $\Delta K_{i j}(t)$ occurring in correspondence with the AE event,

$$
\Delta U_{i j}(t)=\frac{1}{2} x(t)^{2} \Delta K_{i j}(t)
$$

while the dissipated energy $\Delta \Omega_{i j}$ is assumed to be proportional to the newly created crack surfaces $A_{i j}$ :

$$
\Delta \Omega_{i j}=G_{C} A_{i j}
$$

where $G_{C}$ is the critical strain energy release rate of the material. The surfaces $A_{i j}$ are also assigned randomly, according to either a uniform or an inverse power-law distribution, in conformity with the multiscale character of the fracturing process [3].

As discussed elsewhere [23], the model yields results that are consistent with the experimental results from AE tests used to characterize damage progression in materials. In particular, avalanches in fiber ruptures occur, due to the load transfer from broken to intact fibers in the course of loading. Specific distributions for these avalanches are known for different FBMs - namely, an asymptotic power-law distribution with exponent $-5 / 2$-occurring in the majority of cases, although exceptions exist [24,25]. The present model also yields a power-law distribution $P$ for the released $\mathrm{AE}$ energy $T$ :

$$
P(T) \propto T^{\alpha},
$$

with an exponent $\alpha$ which typically varies between -1.6 and -2.1 , depending on the chosen parameters (mainly the chosen Weibull modulus $m$ ). Typical results are shown in Fig. 2 for a Young's modulus of $E=23 \mathrm{GPa}$, a material nominal strength of $\sigma_{0}=10 \mathrm{MPa}, G_{C}=10, N_{x}=5000, N_{y}=1000$, and $m=2$. The scaling exponent here is $\alpha=-1.9$. This and other numerically computed exponents differ from the $-5 / 2$ value, but are closer to the experimental values found in the literature (see [29] and references therein). It has been suggested that this power-law behavior could be indicative of an underlying critical dynamics of the process, related to the absence of a characteristic length and a self-similarity in the microfracturing phenomenon.

As system variable characterizing the damage evolution we now choose $Y(t)=T(t) / T_{\text {tot }}-\mathrm{i}$.e., the kinetic energy $T$ released due to AE at time $t$ divided by the total energy $T_{\text {tot }}$ released in the entire loading cycle. An initial condition of $Y_{0}=0.1$ is chosen to account for preexisting damage in the sample. A specimen with the discretization $N_{x}=1000$ and $N_{y}=100$ is considered, subjected to a linearly increasing load. To evaluate the influence of the Weibull modulus, three

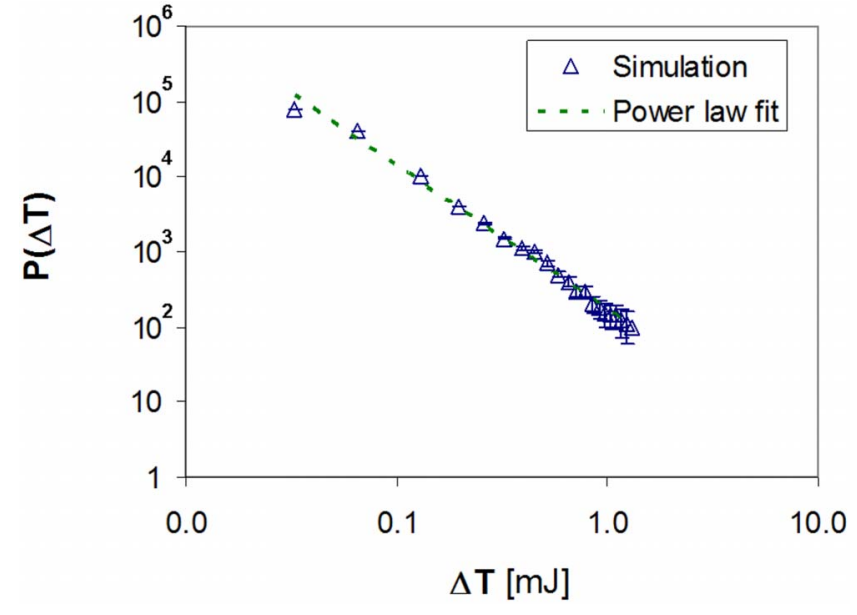

FIG. 2. (Color online) Distribution $P(T)$ of the simulated released AE energy $T$ during damage progression in a virtual specimen. Simulation data include error bars.

different values are considered: $m=2.5, m=1.7$, and $m=0.7$. The results are discussed together with PUN analysis in the following section.

\section{COMPARATIVE ANALYSIS}

Numerical results from Sec. III are analyzed using the PUN approach [14], based on a power-series expansion of the second derivative $\ddot{z}$ of the rescaled system variable with respect to its first derivative $a=\dot{z}$. The stress $\sigma$ is assumed to be the independent variable.

We recall here the basic formalism

$$
\ddot{z}=f(a)=\sum_{n=1}^{\infty} b_{n} a^{n},
$$

where

$$
\begin{gathered}
z(\tau)=\ln \left(\frac{Y}{Y_{0}}\right) / \ln \left(\frac{Y_{\infty}}{Y_{0}}\right), \\
a=\dot{z}(\tau)=\frac{Y}{Y_{0}}\left(\frac{d Y}{d \sigma}\right) /\left(\frac{d Y_{0}}{d \sigma}\right), \\
\tau=\frac{\sigma}{\ln \left(Y_{\infty} / Y_{0}\right)} \frac{1}{Y_{0}}\left(\frac{d Y}{d t}\right)_{0} .
\end{gathered}
$$

and

$$
Y_{0}=Y(0), \quad Y_{\infty}=\lim _{\sigma \rightarrow \infty} Y(\sigma) .
$$

If a satisfactory fit of the experimental data is obtained by truncating the set at the $N$ th term (or power of $a$ ), then we state that the underlying phenomenology belongs to the PUN class UN. In the present context we will limit the discussion to the first three classes U1, U2, and U3, and assume $Y_{\infty}$ $=1$, as discussed in Sec. II.

To determine the lowest class to satisfactorily describe the data, the curve $f(a)$ is plotted as a function of $a$ for each of 

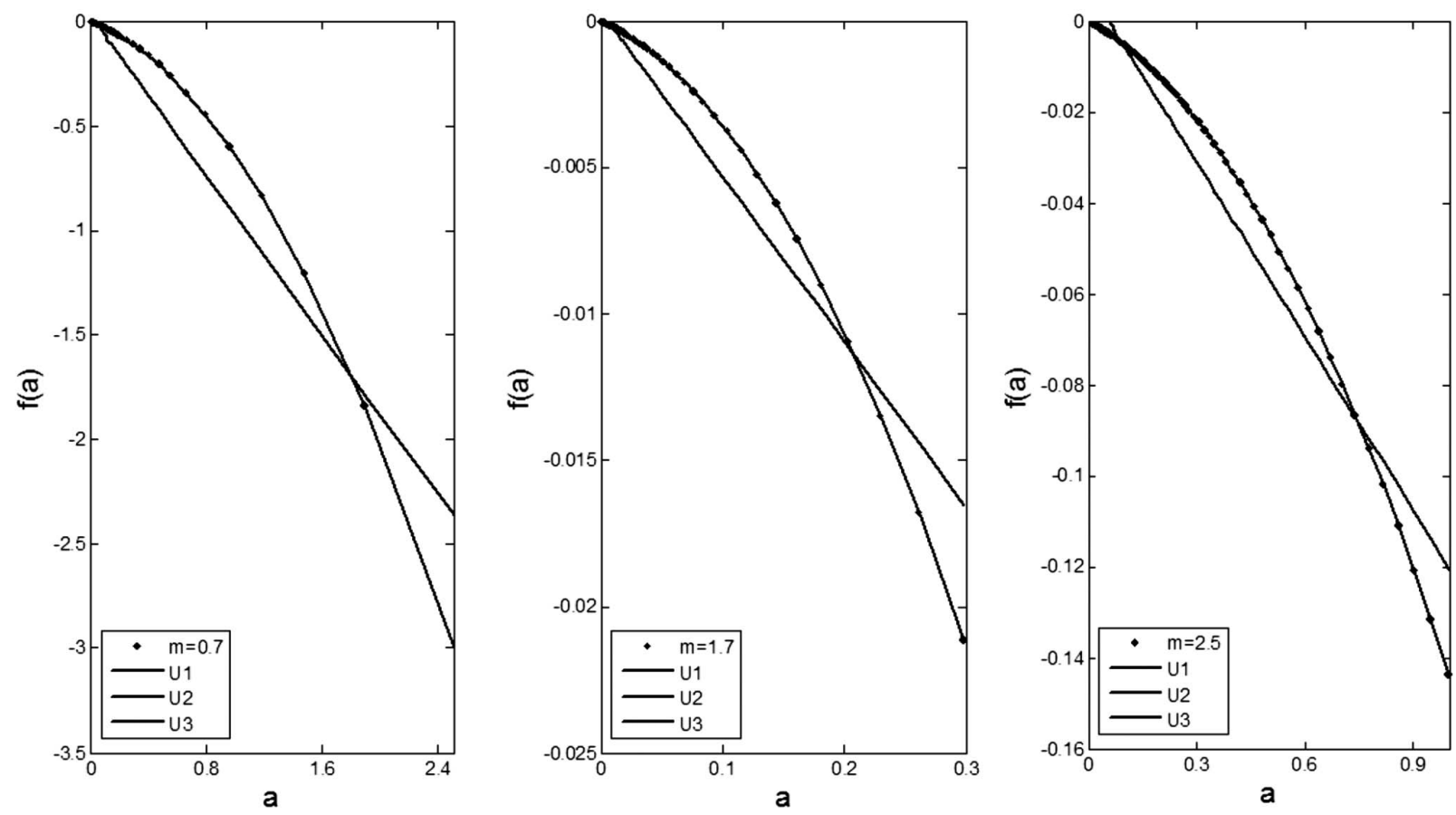

FIG. 3. Comparison among the U1, U2, and U3 fittings of the curve $f(a)$, obtained from the simulation data for the three considered values of the Weibull modulus m. While U2 provides a substantial improvement over U1 (dotted), almost no gain is obtained with U3, in spite of the additional parameter. In fact, in the figures, the U3 are almost indistinguishable from U2 curves.

the three selected values of the Weibull modulus, showing that in each case the class U2 provides an excellent fit to the data obtained by simulation. Indeed, as shown in Fig. 3, in all the three cases the U1 dotted straight line does not adequately fit the curves $f(a)$, while the $\mathrm{U} 2$ line exhibits a very good agreement $\left(R^{2}>0.998\right)$, and there is almost no improvement in going to U3, in spite of the additional parameter included in this class. This confirms the validity of the model presented in Section II.

Let us now use Eq. (6) to fit the curves $Y(\sigma, t)$ obtained numerically as a function of the applied stress. The data in Fig. 4 show that different types of curves are obtained for Weibull modulus values of $m>1$ and $m<1$, respectively. This could be expected, due to the fact that the Weibull distribution becomes monotonically decreasing for $m<1$. Both types of curves can nevertheless be adequately fitted by $\mathrm{U} 2$ curves, with excellent values of $R^{2}$. This again confirms the validity of the proposed damage model.

From the fit it is possible to extract the value of the $p$ parameter. For the three values of $m=2.5,1.7$, and 0.7 we obtain $p=0.90,0.83$, and 0.62 , respectively. The logistic-type behavior of $Y(\sigma, t)$ obtained for $m=0.7$ corresponds to $p$ $<0.67$-i.e., $D<2$ - while the sigmoid-type curves obtained for $m>1$ correspond to $p>0.67$-i.e., $2<D<3$-thus highlighting the relationship between the Weibull modulus and $p$.

\section{CONCLUSIONS}

The PUNs represent a tool for the classification and interpretation of different phenomenologies in the context of cross-disciplinary research. The PUN class U2 is of special
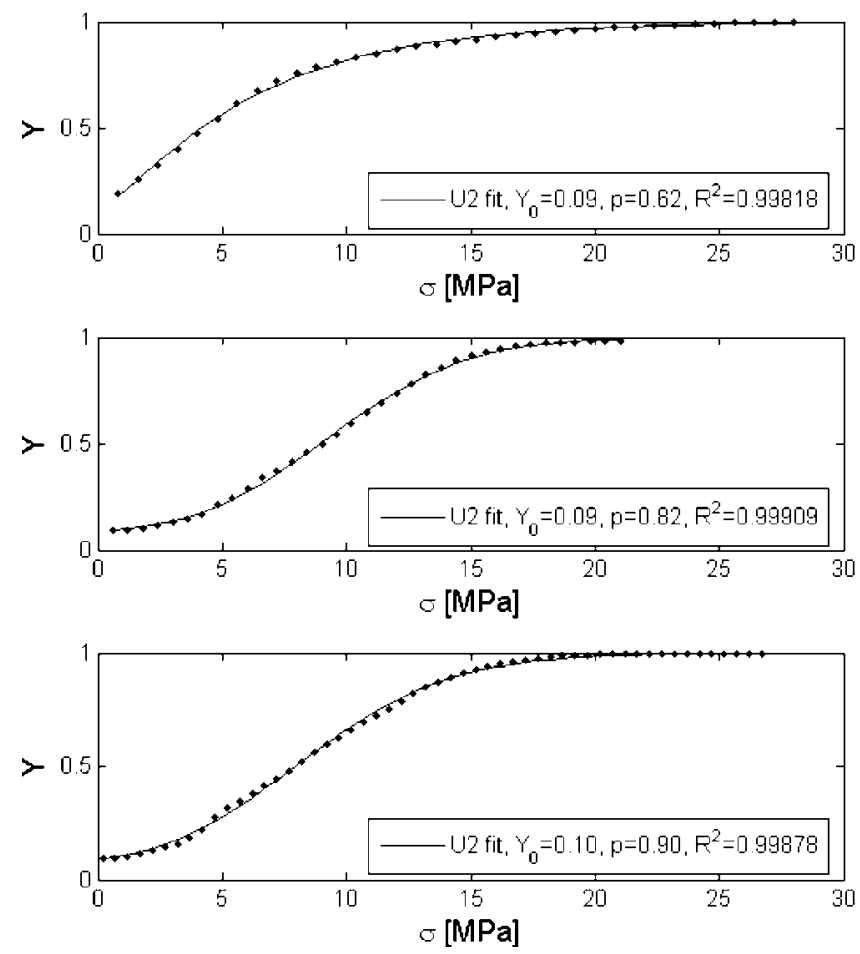

FIG. 4. Simulated damage factor $Y$ (dots) vs applied stress for different values of the Weibull modulus: $m=0.7,1.7$, and 2.5 starting from the top, respectively. The data are fitted with Eq. (6). The excellent values of $R^{2}$ confirm that the analyzed phenomenology belongs to the PUN U2 class. 
relevance, since it includes, as subcases, all growth models proposed to date [30]. As an example of an application of the proposed methodology, we have considered here the study of the damage evolution in a generic physical system subjected to an external stress, which is increasing in time. We have found a correspondence between the probability function, which determines the type of damage evolution, and the exponent $p$ of the $\mathrm{U} 2$ fitting curve.

Given the absolute generality of the approach, our findings give rise to a number of intriguing questions. For example, if we proceed in analogy with the work by West and collaborators in the field of allometric scaling of living organisms [5], can a "universal" damage growth curve be constructed also for materials subject to loading, once the required set of rescaling parameters is known? If this were true, many applications could be envisaged. One possibility could be to exploit the method as a simple tool to verify specimen integrity. In principle, for a given known material, it could be possible to extract only a small part of the curve $Y(\sigma, t)$ and derive the value of $p$. Once the $p$ parameter is known, it is trivial to extract other relevant parameters, such as the critical damage level of the structure under study. Finally, the capability of the method to detect and quantify minute differences among similar-looking data (e.g., between the sigmoidal curves obtained with $m=2.5$ and 1.7) demonstrates its ability to act as a "magnifying glass" in the analysis of experimental data (in this case to evaluate the damage state of a sample).

\section{ACKNOWLEDGMENTS}

A.S.G. wishes to acknowledge the support from the C.R.T. Foundation. F.B. was supported by the "Bando Regionale sulla Ricerca Scientifica Applicata-2004" by Regione Piemonte. N.P. and A.P. are supported by the "Bando Ricerca Scientifica Piemonte-2006”.
[1] J. Lemaitre and R. Desmorat, Engineering Damage Mechanics: Ductile, Creep, Fatigue and Brittle Failures (SpringerVerlag, Berlin, 2005).

[2] M. Scalerandi, M. Nobili, M. Griffa, A. S. Gliozzi, and F. Bosia, Phys. Rev. B 73, 092103 (2006).

[3] A. Carpinteri and N. Pugno, Nature Mater. 4, 421 (2005).

[4] G. Durin and S. Zapperi, Phys. Rev. Lett. 84, 4705 (2000).

[5] S. Zapperi, A. Vespignani, and H. E. Stanley, Nature (London) 388, 658 (1997); F. F. Csikor, C. Motz, D. Weygand, M. Zaiser, and S. Zapperi, Science 318, 251 (2007).

[6] G. B. West, J. H. Brown, and B. J. Enquist, Science 276, 122 (1997).

[7] P. F. Verhulst, Correspondence Mathématique et Physique 10, 113 (1838).

[8] L. Von Bertalanffy, Growth 30, 123 (1966).

[9] B. Gompertz, Philos. Trans. R. Soc. London 123, 513 (1825).

[10] J. H. Brown and G. B. West, Scaling in Biology (Oxford University Press, Oxford, 2000).

[11] G. B. West, J. H. Brown, and B. J. Enquist, Science 284, 1677 (1999).

[12] G. B. West and J. H. Brown, Phys. Today 57(9), 36 (2004).

[13] C. Guiot, P. G. Degiorgis, P. P. Delsanto, P. Gabriele, and T. S. Deisboeck, J. Theor. Biol. 225, 147 (2003).

[14] P. P. Delsanto, C. Guiot, P. G. Degiorgis, A. C. Condat, Y. Mansury, and T. S. Desiboeck, Appl. Phys. Lett., 85, 4225 (2004); C. Guiot, P. P. Delsanto, A. Carpinteri, N. Pugno, Y. Mansury, and T. S. Deisboeck, J. Theor. Biol. 240, 459 (2006).

[15] P. Castorina, P. P. Delsanto, and C. Guiot, Phys. Rev. Lett. 96, 188701 (2006); Universality of Nonclassical Nonlinearity with applications to NDE and Ultrasonics, edited by P. P. Delsanto
(Springer, New York, 2007).

[16] N. Pugno, Med. Hypotheses 69, 441 (2007).

[17] L. M. A. Bettencourt, J. Lobo, D. Helbing, C. Kuhnert, and G. B. West, Proc. Natl. Acad. Sci. U.S.A. 104, 7301 (2007).

[18] L. M. Kachanov, Izv. Akad. Nauk SSSR, Otd. Tekh. Nauk. 8, 26 (1958).

[19] Y. N. Rabotnov, Creep Problems in Structural Members (North-Holland, Amsterdam, 1969).

[20] A. Carpinteri and N. Pugno J. Appl. Mech. 69, 854 (2002).

[21] A. Carpinteri, Mech. Mater. 18, 89 (1994); A. Carpinteri, Int. J. Solids Struct. 31, 291 (1994).

[22] A. Carpinteri and N. Pugno, Int. J. Numer. Analyt. Meth. Geomech. 26, 499 (2002)

[23] F. Bosia, N. Pugno, G. Lacidogna, and A. Carpinteri, Int. J. Solids Struct. 45, 5856 (2008); N. Pugno, F. Bosia, and A. Carpinteri, Small 4, 1044 (2008).

[24] M. Kloster, A. Hansen, and P. C. Hemmer, Phys. Rev. E 56, 2615 (1997).

[25] R. C. Hidalgo, F. Kun, and H. J. Herrmann, Phys. Rev. E 64, 066122 (2001).

[26] P. Bhattacharyya, S. Pradhan, and B. K. Chakrabarti, Phys. Rev. E 67, 046122 (2003).

[27] F. Kun, S. Zapperi, and H. J. Herrmann, Eur. Phys. J. B 17, 269 (2000).

[28] D. De Tommasi, G. Puglisi, and G. Saccomandi, Phys. Rev. Lett. 100, 085502 (2008).

[29] A. Petri, G. Paparo, A. Vespignani, A. Alippi, and M. Costantini, Phys. Rev. Lett. 73, 3423 (1994).

[30] De Vladar, J. Theor. Biol. 238, 245 (2006). 